\title{
Familiaridad de marca: Crítica al concepto actual y nueva propuesta de una composición multidimensional
}

Lizardo Vargas Bianchi | lizardo.vargas@udep.pe

Unidad de Investigación en Comunicaciones de Marketing (UICM), Universidad de PiURA

Resumen: En la literatura se ha establecido el concepto de familiaridad de marca como una construcción unidimensional, compuesta sólo por factores cognitivos. Con base en esa concepción se han desarrollado distintos estudios sobre la familiaridad y su carácter moderador sobre diversos efectos en el comportamiento del consumidor. El artículo presenta dos hipótesis críticas a la perspectiva unidimensional del concepto y plantea una composición multidimensional del mismo. Se realiza una argumentación teórica y un estudio cualitativo de jóvenes consumidores de máquinas de afeitar. Los resultados exponen la validez de una composición multivariable de familiaridad.

Palabras clave: Familiaridad de marca, procesamiento de información, conocimiento, awareness

Abstract: In academic literature the concept of brand familiarity has been accepted as a unidimensional construct, composed by cognitive factors. Based on this conception several studies have been developed, mostly on the moderating effect of familiarity over different aspects of consumer behavior. This article presents two hypothesis criticising the unidimensional view of the concept, as well as a multidimensional understanding of it. The paper presents theoretical argumentation and a qualitative study of razor blades consumption among a convenience sample of young males. Results support the understanding familiarity as a multivariable construct.

Key words: Brand familiarity, information processing, knowledge, awareness 


\section{Introducción}

Los estudios que versan sobre familiaridad de marca la muestran como una variable que se halla presente, de una u otra manera, en distintas etapas que componen la dinámica comercial de una marca, desde los procesos cognitivos y emocionales que intervienen en la toma de decisión, hasta el desarrollo de los mensajes y estímulos publicitarios de un producto (Vargas, 2006).

Sin embargo, esta variable suele abordarse de forma tácita cuando se trata sobre el comportamiento y psicología del consumidor. El hecho de actuar en ausencia de notoriedad quizá debido a la simplicidad propia del concepto-, puede originar que los efectos de la familiaridad sean dados por supuesto y en consecuencia no ser observados con detenimiento.

No obstante, la cobertura que ha recibido en la literatura, sólo un grupo de autores (Baker, Hutchinson, et. al., 1986; Alba y Hutchinson, 1987) ha propuesto una definición del concepto 'familiaridad', aceptada de modo general desde que fue publicada. En el presente artículo se realiza una crítica al contenido de la definición del término, y se procura demostrar que el significado otorgado al mismo puede poseer un mayor grado de precisión.

Para ello primero se presenta una argumentación teórica y luego un estudio cualitativo del comportamiento de compra y uso de máquinas de afeitar con una muestra de conveniencia $(n=24)$ de jóvenes entre 18 y 23 años, con el objeto de identificar posibles correlaciones entre la familiaridad, el conocimiento y otras variables no cognitivas.

\section{Antecedentes y revisión de la literatura}

La primera referencia hacia la familiaridad, enmarcada en un contexto comercial, la realizan Bogart y Lehman (1973). Los autores observaron que los productos comprados con mayor frecuencia y beneficiados con fuerte apoyo publicitario mostraban más familiaridad entre los individuos, en el simple sentido de ser mejor conocidos por éstos.

Los investigadores no diferenciaron dicho efecto de la variable awareness -entendida como conciencia o identificación de una marca-, factor que, como la familiaridad, también aumenta luego de la exposición publicitaria ya que se encuentra ligada al grado de conocimiento. Expresado de otra forma, Bogart y Lehman (1973) no formularon una diferencia semántica entre ambos conceptos: awareness y familiaridad.

Posteriormente, Politz (1975) sostuvo que la confianza originada al publicitar una marca era una condición suficiente para originar confianza hacia la misma. El autor estableció una cierta equivalencia entre confianza y familiaridad, denominó el efecto como el 'Principio de Familiaridad'. Aunque no lo definió, parece plausible sostener que también pudo nombrarse 
'Principio de Confianza', sin menoscabar el sentido que el autor procuraba. En último término, y de modo similar a Bogart y Lehman (1973), la utilización de la palabra 'familiar' traía consigo la connotación de aquello conocido, lo que no resulta ajeno, remoto o poco definido por el consumidor.

Los autores Park y Lessig (1977) utilizaron el término familiaridad con base en estudios relacionados con el esfuerzo cognitivo en la toma de decisiones, una aproximación más cercana a la psicología cognitiva. Ambos investigadores se fundaron en las categorías de resolución de problemas y análisis de proceso de toma de decisiones introducidas por Howard y Sheth (1969). Éstos últimos sugirieron, en breve, tres escenarios donde se observan procesos cognitivos y comportamentales con características particulares en toma de decisión de un individuo: básicamente la información a la que tiene acceso, los pasos que sigue para decidir y el tiempo que toma el procedimiento. Estas fases y las siglas por las que son conocidas (correspondientes al inglés originario) son la Solución Extensiva de Problemas (EPS), Solución Limitada de Problemas (LPS), Solución Rutinaria de Problemas (RPS) y Aburrimiento o Solución Exploratoria de Problemas (SEP).

Park y Lessig (1977) sostuvieron que las fases del proceso de toma de decisión dan origen una curva, originada en función a la información que el consumidor posee y lo habilita a tomar una decisión apropiada, la que denominaron 'curva de familiaridad'.

En definitiva, la curva de familiaridad expresa la relación entre el grado de información, la consecuente disminución en la ambigüedad del consumidor hacia la marca y la velocidad en la decisión. Como se sostuvo al inicio, parece factible considerar el uso del término familiaridad enmarcado en una dinámica cognitiva, donde se pone de manifiesto una relación entre el nivel de conocimiento, procesamiento de información y el comportamiento de decisión-compra.

Algunos años más tarde, Park y Lessig (1981), en un paper sobre sesgos y heurística en la toma de decisión de compra, plantearon una distinción en el sentido de familiaridad. En primer lugar, referido a cuánto sabe la persona sobre el producto; por otro lado, en función a cuánto cree la persona saber sobre el producto. Los autores llamaron a la primera 'familiaridad objetiva' y a la segunda 'familiaridad subjetiva'.

Es posible haber utilizado otra terminología, ya que en último término el nivel de información y su utilidad para la toma de decisión, (es decir la familiaridad originada por cuánto se sabe del producto), no tiene un baremo absoluto y en consecuencia ésta siempre tiene carácter individual o subjetivo. No obstante puede resultar de interés para mediciones de tensión de compra en categorías de productos complejos para cuya elección se requiere mayor conocimiento.

Johnson y Russo (1984) introdujeron otra perspectiva en el estudio de la familiaridad, al argumentar la participación de esta variable en la dinámica cognitiva del individuo. Los autores observaron que la familiaridad hacia un objeto ejercía influencia en el aprendizaje de 
nueva información sobre el mismo. Afirmaron que el conocimiento previo aumenta la habilidad del consumidor de codificar y rememorar nueva información. Ante esta mejora en la codificación, los consumidores familiarizados desarrollan un incremento en la capacidad de organizar nuevo conocimiento sobre la marca.

Este efecto se registró como la función moderadora o moduladora de la familiaridad, donde se presenta la variable como factor que participa en la regulación de los diversos esfuerzos cognitivos de la persona. La función moderadora es quizá el aspecto de la familiaridad sobre el que más estudios se han publicado hasta el momento (Gill, 1988; Ambler, Braeutigam, et.al., 2004; Ha, 2005; Aurier, Jean y Zaichowsky, 2000; Kent y Allen, 1994; Vanhuele, 1999; Kent y Kellaris, 2001; Dimofte y Yalch, 2008; Phelps y Thorson, 1991; Simonin y Ruth, 1998; Campbell y Keller, 2003; Machleit y Wilson, 1988; Dahlén y Lange, 2004; Hardesty, Carlson y Bearden, 2002; Sun, Lin, et. al., 2009).

Sin embargo, la familiaridad de marca no fue definida hasta la publicación de un artículo referido a la vinculación entre la publicidad y la toma de decisiones a partir de conjuntos de consideración (consideration sets/evoked sets, Howard y Sheth (1969)- por los autores Baker, Hutchinson, et. al. (1986).

En el artículo manifestaron su intención de presentar una definición que vinculase de forma directa la familiaridad con el conocimiento - propuesta razonable cuando se examina la literatura precedente-, y que fuera de carácter pragmático, con el fin de facilitar su investigación: estudiar el efecto moderador del conocimiento (familiaridad) sobre diversos aspectos de la decisión de compra, como el reconocimiento, la recordación, la preferencia de marca o las actitudes del consumidor.

Con ello en mente y desde el inicio de su artículo, Baker, Hutchinson et al. equipararon los términos familiaridad y awareness, a partir de ello propusieron una definición de familiaridad:

la familiaridad marca es una construcción unidimensional que se encuentra directamente relacionada a la cantidad de tiempo que se ha pasado procesando información sobre la marca, con independencia del tipo de contenido que haya sido procesado (1986: 637).

Aunque el objetivo de los autores no era definir el concepto de familiaridad, sino observar los efectos del conocimiento en las elaboraciones cognitivas que suceden antes y después de la compra, el enunciado de Baker, Hutchinson et al. (1986) se ha convertido en la definición estándar del término familiaridad en la literatura académica.

\section{Hipótesis}

Como se ha hecho mención anteriormente, la propuesta de Baker, Hutchinson, et. al. (1986) fue establecida con un objetivo funcional: colocar un nombre a la construcción awarenessconocimiento de marca. Los propios autores describieron el constructo en los siguientes términos: "la forma más rudimentaria de conocimiento por parte del consumidor" (p.637). 
Sin embargo, el propio artículo despliega el uso del concepto familiaridad en diversos planos, por ejemplo conjuntos de consideración, reconocimiento y preferencia de marca, recordación y claves de recordación, el efecto de exposición y el efecto moderador, entre otros.

La simplificación del término, como lo realizan Baker, Hutchinson et al. (1986), puede facilitar la flexibilidad y conveniencia en su uso. No obstante, diversos estudios sobre familiaridad (muchos de ellos citados en el recuento de la literatura) manifiestan las distintas aplicaciones que contiene el término.

En este sentido, no parece razonable circunscribir como una construcción unidimensional la conceptualización, estudio y utilización de la familiaridad de marca. En orden con esta idea se crítica la propuesta de Baker, Hutchinson et al. (1986) y se postulan dos hipótesis:

H1: la familiaridad de marca no es una es una construcción unidimensional, sino multidimensional.

H2: familiaridad de marca no es sinónimo de awareness respecto a una marca.

\section{Argumentación teórica}

En cuanto a H1, Baker, Hutchinson et al. (1986) justifican el uso de familiaridad como una expresión práctica para contener el sentido de conocimiento y awareness del consumidor respecto a la marca. La unidimensionalidad que sostienen los autores es precisamente el confinamiento estricto de la familiaridad al plano del conocimiento, de la información poseída por un consumidor hacia una marca. Podría afirmarse que comprenden la familiaridad como una ausencia de ignorancia o una ignorancia parcial.

En este sentido, un individuo que haya procesado suficiente información para lograr reconocer una marca, es un individuo que ha superado la ignorancia total o parcial, y a partir de ese momento afirmar que la marca le resulta familiar.

Sin embargo, es posible observar distintos grados de conocimiento de un consumidor hacia un producto. Por ejemplo, una persona que sabe de la existencia de marca de conservas, aunque nunca la haya visto, ni tampoco tenido la oportunidad de consumirla, en la lógica de Baker, Hutchinson et al. (1986) podría decirse que dicha persona se encuentra familiarizada con la marca.

El ejemplo describe el nivel más básico de conocimiento que se puede distinguir en una marca, el reconocimiento. Sin embargo, el consumidor está en capacidad de ir más allá de este grado, al entrar en contacto con el producto y tener información por haberlo utilizado, por la publicidad, o por los comentarios de terceras personas. 
Si bien es plausible sostener que tanto en el reconocimiento como en el conocimiento existe familiaridad, no parece razonable negar una variación gradual en el factor.

Puede suceder que la imprecisión conceptual de Baker, Hutchinson et al. (1986) se origina cuando simplifican el sentido del conocimiento a tal nivel que no deja espacio a que este factor evolucione en un proceso de aprendizaje (ver Hayes-Roth, 1977) y coexista con otros factores que también participan del comportamiento del consumidor.

Es precisamente la evolución y coexistencia con diversos factores lo que origina una pluralidad en las dimensiones de la familiaridad (ver Ha 2005). Si bien ésta se inicia en un plano cognitivo, en su evolución puede expandirse hacia los planos afectivos y conativos. La acción de la familiaridad en estas tres dimensiones se pone de manifiesto en las siguientes relaciones entre el individuo y el producto:

Dimensión A: Mejora de la habilidad cognitiva

La familiaridad se inicia con el conocimiento del producto, el cual puede tener un aumento cualitativo gradual. Es posible identificar niveles de conocimiento que van desde el simple reconocimiento de la marca, hasta un grado alto de pericia en la utilización del producto, derivado de consumos precedentes repetidos.

Se ha reportado la existencia de una correlación positiva entre el grado de conocimiento que se tiene hacia una marca y la facilidad para procesar información, de manera particular información nueva (Biswas, 1992; Coates, Butler, Berry, 2006). Es decir, la familiaridad reduce el esfuerzo cognitivo en tomas de decisión posteriores. Por ejemplo, nuevas presentaciones del producto, cambios en su empaque, o nuevos usos sugeridos por la publicidad.

Dimensión B: Habilidad operativa

De la misma manera como la familiaridad favorece el desempeño cognitivo, al moderar el esfuerzo cognitivo requerido para procesar información, la familiaridad también contribuye a que el individuo pueda utilizar o manejar el producto con mayor pericia (Alba y Hutchinson, 1987).

Se advierte la misma correlación positiva (Alba y Hutchinson, 1987) que en el punto anterior, donde el consumidor más familiarizado con el producto muestra más destreza en su relación con el mismo. La utilización reiterada durante periodos de tiempo, logra que la persona sepa utilizar un producto con mayor habilidad y provecho. El tiempo y conocimiento requeridos se encontrarán en proporción directa a la complejidad del mismo y a la destreza propia de la persona. Por ejemplo, mediante la utilización repetida de una máquina de afeitar, el usuario consigue identificar los movimientos apropiados, las oportunidades de lavado necesarios, el momento de descartar la máquina, con el objeto de obtener una afeitada limpia y sin irritar la piel de la cara. 
De esta forma, se puede sostener que la familiaridad tiene una repercusión comportamental en la actuación del consumidor. Nuevamente se exhibe la coexistencia del conocimiento con otra variable, en este caso conativa, y es posible encontrar un vínculo. A mayor conocimiento, mejor habilidad en las acciones de uso y utilidad en los atributos del producto.

Dimensión C: La valoración de producto y sus atributos

Como se ha mencionado en párrafos anteriores, en la base de la construcción de la familiaridad se encuentra el conocimiento del producto, el cual se logra procesando información de diversa fuentes.

La dinámica de procesamiento de información del producto, es decir, mantener nexos de diverso tipo con la marca y de forma regular en el tiempo, igualmente faculta a que el consumidor desarrolle vínculos valorativos hacia la marca: algunos productos serán preferidos ante otros. La preferencia no es estrictamente una construcción cognitiva, sino que también revela aspectos afectivos hacia el objeto con el cual se ha mantenido un vínculo.

La valoración se exhibe cuando en el consumidor se observa una empatía o gusto por una marca en particular (Monroe, 1976), y en cierto modo explica la conformación de conjuntos de consideración para facilitar la compra (Nedungadi, 1990; Kardes, Kalyanaram, et. al., 1993). Estos conjuntos consolidan las marcas que son más valoradas, las que gozan del afecto y cercanía del consumidor; el conjunto evocado o de consideración va más allá del simple conocimiento del producto.

\subsection{Metodología y estadística para la argumentación teórica}

Para sustentar la argumentación teórica precedente, referida a que la familiaridad es una variable compuesta por factores adicionales al cognitivo, se realizó un estudio entre consumidores de máquinas de afeitar. El objeto del estudio fue registrar variables del comportamiento de compra y uso del producto para observar la posible relación entre el conocimiento y los factores conativos y afectivos.

El estudio se realizó a una muestra de conveniencia $(n=24)$ no probabilística, de jóvenes varones en edades comprendidas entre los 18 y 23 años, estudiantes universitarios de la ciudad de Lima, pertenecientes a niveles socioeconómicos homogéneos. La muestra de conveniencia fue compuesta por una selección aleatoria entre decisores de compra y usuarios de máquinas de afeitar. Se realizó durante el mes de octubre.

Se definió la categoría máquinas de afeitar por contar con una serie de características que facilitaban la medición de las variables exploradas en los consumidores. La categoría está compuesta por una amplia oferta, en la que se observan diferencias utilitarias y de precio - 
siempre accesible al público estudiado- y fácil acceso en puntos de venta. Es un producto de uso frecuente, baja complejidad funcional y con baja percepción de riesgo económico y personal.

La herramienta utilizada para recolectar la información fueron encuestas individuales asistidas, constituidas por preguntas estructuradas y de respuesta cerrada. La encuesta, de ocho preguntas, estuvo diseñada para determinar el grado de conocimiento, experimentación, satisfacción y vinculación de los individuos muestrales con sus marcas de máquina de afeitar de uso actual.

Para identificar la experiencia de uso y conocimiento del producto se preguntó a los individuos su edad actual, la edad desde que se afeitan y la edad en que identificaron la marca y modelo de máquina de afeitar que mejor cumplía con sus expectativas. Asimismo, se les preguntó por su actual frecuencia semanal media de afeitado.

Para registrar las respuestas a las preguntas valorativas -satisfacción y vinculación con la marca- se les inquirió sobre su grado de aceptación de desempeño de la máquina de afeitar actual y si recomendarían esa misma marca de máquina de afeitar a otra persona.

Para registrar las respuestas a estas preguntas, los encuestados debían otorgar una puntuación dentro de una escala unipolar de Likert (donde 1 era la menor puntuación y 5 la máxima) a cada pregunta.

Como pregunta de filtro para validar la coherencia de ambas respuestas, también se preguntó a cada individuo si había pensando cambiar de marca de máquina de afeitar durante el último mes. En este caso la respuesta debía corresponder a un campo dicotómico sí/no.

\section{Resultados y discusión}

La población de la muestra de conveniencia tuvo las siguientes características etárias (Tabla 1):

Tabla 1. Características etárias de la población estudiada

\begin{tabular}{|l|l|l|l|l|l|l|l|}
\hline N & Media & Mediana & Moda & Mínima & Máxima & $\begin{array}{l}\text { Desviación } \\
\text { estándar }\end{array}$ & Varianza \\
\hline 24 & 19.58 & 19 & 19 & 18 & 23 & 1.34 & 1.81 \\
\hline
\end{tabular}

En cuanto a la experiencia de uso y frecuencia de la muestra, se preguntó a los encuestados la edad en que empezaron a afeitarse, la edad en que definieron el tipo y marca de máquina de afeitar de su preferencia, y la frecuencia ordinaria semanal de afeitado. Estos resultados se exhiben en la Tabla 2. Con esa información es posible concluir el grado de conocimiento por experiencia directa que los individuos tienen del producto y marcas concretas. 
Tabla 2. Experiencia y frecuencia de uso

\begin{tabular}{|l|l|l|l|}
\hline N=24 & $\begin{array}{l}\text { Dimensão } \\
\text { dos gru }\end{array}$ & Sexo & Idade \\
\hline Media & 12.3 & 17.4 & 2.37 \\
\hline Mediana & 15 & 17.5 & 2.5 \\
\hline Moda & 15 & 17.19 & 3 \\
\hline $\begin{array}{l}\text { Desviación } \\
\text { estándar }\end{array}$ & 1.72 & 1.52 & 0.92 \\
\hline
\end{tabular}

La población observada mostró un media de 4.3 años de uso de máquinas de afeitar, y una media de 2.2 años de uso desde que han definido cuál es la máquina ofertada en el mercado que mejor cumple con sus expectativas de desempeño.

Si bien la media de uso actual semanal del producto es relativamente baja, 2.4 veces por semana, la cantidad de tiempo en años de experiencia del producto compensa esa asimetría y permite afirmar que los consumidores encuestados tienen un nivel razonable de experiencia y conocimiento del producto. Los consumidores estudiados llevan un media de 4.3 años utilizando máquinas de afeitar, y un media de 2.2 años comprando aquella que han identificado como la más apropiada.

Respecto a la satisfacción y vinculación que los consumidores muestran hacia las máquinas de afeitar de su elección, se preguntó a los encuestados que otorgaran una puntuación de 15 en una escala unipolar de Likert a las preguntas: qué te parece el desempeño de la máquina de afeitar que utilizas, y con qué intensidad recomendarían esa máquina a una tercera persona. La Tabla 3 exhibe los datos obtenidos.

Tabla 3. Satisfacción de uso

\begin{tabular}{|l|l|l|}
\hline N=24 & $\begin{array}{l}\text { Percepción de } \\
\text { desempeño de la } \\
\text { marca preferida } \\
(1-5)\end{array}$ & $\begin{array}{l}\text { Grado de } \\
\text { recomendación } \\
\text { de la marca } \\
\text { preferida a otra } \\
\text { persona (1-5) }\end{array}$ \\
\hline Media & 3.95 & 3.75 \\
\hline Mediana & 4 & 4 \\
\hline Moda & 4 & 4 \\
\hline $\begin{array}{l}\text { Desviación } \\
\text { estándar }\end{array}$ & 0.75 & 0.98 \\
\hline
\end{tabular}

La información manifiesta un alto nivel de satisfacción con las marcas elegidas por los consumidores (media +3.95 , en una valoración de 1 a 5 ), que a su vez se corresponde con 
el nivel de recomendación de marca que harían a terceras personas (media +3.8 , en una valoración de 1 a 5).

Asimismo, se calculó el coeficiente de correlación de Pearson entre la 'Percepción del desempeño de la marca preferida' y el 'Grado de recomendación de la marca preferida a otra persona', al cual mostró un resultado alto: $r(22)=0.8, p=.003$, a dos extremos. Estos datos validan el nivel de satisfacción de los consumidores en la elección y utilización de sus marcas de elección.

Finalmente, se preguntó a los encuestados si habían evaluado la posibilidad de cambiar de marca de máquina de afeitar a lo largo del mes precedente. Sólo el 8.3\% había ponderado la posibilidad, dato que valida la satisfacción percibida hacia la marca seleccionada por cada individuo.

En cuanto a H2. Tradicionalmente el vocablo awareness es utilizado para diagnosticar el hecho de si una marca es conocida o no, es decir si ésta se encuentra en la memoria y puede ser evocada, bien de forma espontánea o de modo asistido (Hoyer y Bown, 1990; Laurent, Kapferer, Roussel, 1995; Percy y Rossiter, 2006).

En un sentido estricto, cabe abreviarse el término como un fenómeno de identificación o reconocimiento de la marca por parte del individuo. De hecho en las investigaciones destinadas a medir el grado de awareness la pregunta que se realiza al grupo de estudio es simplemente qué marcas recuerda de una categoría en particular u otorgando pistas para facilitar el recuerdo.

De esta manera, el concepto en sí es dicotómico: una marca se reconoce o no se reconoce. Parece razonable sostener que en la dinámica del awareness sólo entran en acción las variables identificación, memoria y objeto de recuerdo.

Tal es así que la unidimensionalidad parece más propia de la variable awareness que de la familiaridad hacia la marca. Cabe la posibilidad de que aquí radique el origen en la imprecisión conceptual del término familiaridad, dado que los autores Baker, Hutchinson et al. (1986), desde el inicio de su estudio, redujeron el sentido de familiaridad al de awareness, llevándolo consigo hacia un plano unidimensional.

\section{Conclusiones y límites del estudio}

La argumentación teórica, sustentada por el estudio sobre consumo y valoración de máquinas de afeitar en varones jóvenes, parece indicar que la familiaridad hacia una marca no se limita únicamente al plano cognitivo de un producto, sino que tiene consecuencias en la acción y valoración del mismo.

En el estudio realizado se observa que los individuos se acogen a una marca determinada luego de un cierto lapso temporal. El tiempo que se dedica a la experimentación con una 
marca y sus competidores encuentra proporción con el conocimiento que se va logrando respecto a la misma, hasta el momento en que el individuo identifica una alternativa que satisface su utilidad esperada.

Por otra parte, también se pone de manifiesto que cuando la marca es conocida y experimentada, el consumidor puede desarrollar un vínculo valorativo estable hacia ella, es decir una preferencia o gusto hacia una marca antes que otra. Este hecho se observa, en el estudio realizado, en el bajo índice de intención de cambio de marca y el alto grado de puntuación en la recomendación de la marca preferida a otra persona.

A lo largo del tiempo de uso, parece que el consumidor identifica el producto que mejor se adecúa a su grado de utilidad esperada y sobre ello elabora valoraciones y preferencias. Como se sostuvo en la argumentación teórica, la preferencia no es necesariamente una construcción cognitiva ni tampoco una simple identificación o reconocimiento del producto entre sus pares (awareness), sino que se aproxima más al plano subjetivo y emocional.

De esta forma, la información presentada apoya la propuesta de que la familiaridad de marca no solo es un constructo unidimensional, sino multidimensional. Es decir que en su constitución participan factores cognitivos que pueden, posteriormente, desenvolverse y coexistir con otras variables comportamentales y afectivas.

Es importante tener en consideración que el estudio se basa en muestra de conveniencia, y por lo tanto usa una metodología no probabilística. De modo que la información obtenida tiene carácter indicativo y no normativo. Sin embargo es muy probable que los resultados en una muestra validada no difieran de los obtenidos, dadas las variables estudiadas, el patrón de respuesta observado en los individuos seleccionados, así como el cuerpo de conocimiento investigado sobre este aspecto.

Finalmente, no se sugiere necesario cambiar la definición de Baker, Hutchinson et al. (1986), salvo por el término 'unidimensional' (reemplazándolo por 'multidimensional'), ya que sí se observa que el desarrollo y coexistencias de las variables que componen la familiaridad se encuentran relacionadas con el tiempo de procesamiento de información sobre una o varias marcas, y con independencia del tipo o fuente de información que fuera procesada. 


\section{REFERENCIAS BIBLIOGRÁFICAS}

ALBA, J. W., y HUTCHINSON J. W. (1987): "Dimensions of consumer expertise", en Journal of Consumer Research, vol. 13, n. 4, pp. 411-545.

AMBLER, T., BRAEUTIGAM, S., STINS, J., ROSE, S., SWITHENBY, S. (2004): "Salience and choice: neural correlates of shopping decisions", en Psychology and Marketing, vol. 21, n 4, pp. 247-261.

AURIER, P., JEAN, S., y ZAICHOWSKY, J. L. (2000): "Consideration set size and familiarity with usage context", en Advances in consumer Research. vol. 27, pp. 307-313.

BAKER, W., HUTCHINSON, W. J., MOORE, D., NEDUNGADI, P. (1986): "Brand familiarity and advertising: effects on the evoked set and brand preference", en Advances in Consumer Research, Ed. Richard J. Lutz. vol. 13, Issue 1, pp. 637-643.

BISWAS, A. (1992): "The moderating role of brand familiarity in reference price perceptions", en Journal of Business Research, vol. 25, Issue 3, pp. 251262.

BOGART, L., y LEHMAN, C. (1973): "What makes a brand name familiar?", en Journal of Marketing Research, vol. 10, pp. 17-22.

CAMPBELL, M. C., y KELLER, K. (2003): "Brand familiarity and advertising repetition effects", en Journal of Consumer Research, September, vol. 30, Issue 2, pp. 292-304.

COATES, S., BUTLER, L. T., BERRY, D. C. (2006): "Implicit memory and consumer choice: the mediating role of brand familiarity", en Applied Cognitive Psychology, vol. 20, pp. 1101-1116.

DAHLEN, M., y LANGE, F. (2004): "To challenge or not to challenge: Ad-Brand incongruency and brand familiarity", en Journal of Marketing Theory and Practice, vol. 12, n० 3, pp. 20-35.

DIMOFTE, C., y YALCH, R. (2008): "The role of product category familiarity in selfreferent advertising", en Advances in Consumer Research, vol. 35, pp. 726727.

GILL, J. D., GROSSBART, S., LACZNIAK, R. N. (1988): "Influence of involvement, commitment and familiarity on brand beliefs and attitudes of viewers exposed to alternative ad claim strategies", en Journal of Advertising, vol. $17, n^{\circ} 1$, pp. 33-43.

HA, Hong-Youl (2005): "Effects of consumer perceptions of brand experience on the web: Brand familiarity, satisfaction and brand trust", en Journal of Consumer Behavior, vol. 4, pp. 438-452. 
HARDESTY, D. M., CARLSON, J. P. y BEARDEN, W. O. (2002): "Brand familiarity and invoice price effects on consumer evaluations: the moderating role of skepticism toward advertising", en Journal of Advertising, vol. 31, n 2, pp. $1-15$.

HAYES-ROTH, B. (1977): "Evolution of Cognitive Structures and Processes", en Psychological Review, vol. 84, pp. 260-78.

HOWARD, J. A., y SHETH, J. N. (1969) "The Theory of Buying Behaviour". Nueva York, John Wiley \& Sons Inc.

HOYER, W. D., y BROWN, S. P. (1990): "Effects of Brand Awareness on Choice for a Common, Repeat-Purchase Product", en Journal of Consumer Research, vol. 17, pp. 141-148.

JOHNSON, E, J., y RUSSO, E. J. (1984): "Product familiarity and learning new information", en Journal of Consumer Research, vol. 11, n 1, pp. 542-550

KARDES, Frank R., KALYANARAM, G., CHANDRASHEKARAN, M. y DORNOFF, R. J. (1993): "Brand Retrieval, Consideration Set Composition, Consumer Choice, and the Pioneering Advantage", en Journal of Consumer Research, vol. 20, $\mathrm{n}^{\circ} 1$, pp. 62-75.

KENT, R, J., y KELLARIS, J. J. (2001): "Competitive interference effects in memory for advertising: are familiar brands exempt?", en Journal of Marketing Communications, vol. 7, n³, pp. 159-169.

KENT, R. J., y ALLEN, C. T. (1994): "Competitive interference effects in consumer memory for advertising the role of brand familiarity", Journal of Marketing, vol. 58, pp. 97-105.

LAURENT, G., KAPFERER, J-N, ROUSSEL, F. (1995): "The Underlying Structure of Brand Awareness Scores", en Marketing Science, vol. 14, n 3, pp. 170-179.

MECHLEIT, K. A., y WILSON, D. A. (1988): "Emotional feelings and attitude toward the advertisement: the roles of brand familiarity and repetition", en Journal of Advertising, vol. 17, $\mathrm{n}^{\circ}$ 3, pp. 27-35.

MONROE, K. E. (1976): "The influence of price differences and brand familiarity on brand preferences", en Journal of Consumer Research, vol. 3, n 1, pp. 4249.

NEDUNGADI, P. (1990): "Recall and Consumer Consideration Sets: Influencing Choice without Altering Brand Evaluations", en Journal of Consumer Research, vol. 17, n³, pp. 263-276.

PARK, C. W., y LESSIG V. P. (1977): "Judgmental rules and stage of the familiarity curve; promotional implications" en Journal of Advertising, vol. 6, Issue 1, pp. 10-16. 
PARK, C. W., y LESSIG, V. P. (1981): "Familiarity and its impact on consumer decision biases and heuristics", en Journal of Consumer Research, vol. 8, pp. 223-230.

PERCY, L., y ROSSITER, J. R. (2006): "A model of brand awareness and brand attitude advertising strategies", en Psychology and Marketing, vol. 9, Issue 4, pp. 263-274.

PHELPS, J., y THORSON, E. (1991): "Brand familiarity and product involvement effects on the attitude toward an ad brand attitude relationship", en Advances in Consumer Research, vol. 18, pp. 202-209.

POLITZ, A. (1975): "The function of advertising and its measurements", en Journal of Advertising, vol. 4, Issue 2, pp. 10-12.

SIMONIN, B. L., y RUTH, J. A. (1998): "Is a company known by the company it keeps? Assessing the spillover effects of brand alliances on consumer brand attitudes", en Journal of Marketing Research, vol. 35, pp. 30-42.

SUN, Ya-Chung, LIN, Chien-Huang, CHENG, Yin-Hui y CHUANG, Shih-Chieh (2009): "The moderating effect of product familiarity on the endowment effect", en Advances in Consumer Research, vol. 36, pp. 974-975.

VANHUELE, M. (1995): "Why familiar stimuli are better liked. A study on the cognitive dynamics linking recognition and mere exposure effect", en Advances in Consumer Research, vol. 22, pp. 171-175.

VARGAS, L. (2006): "El desarrollo del concepto de familiaridad de marca, su relación con el procesamiento de información realizado por los consumidores y su uso actual como una variable moderadora", en Información Pública, vol. $4, n^{\circ} 2$, pp. 287-239.

[Recibido: 8 de noviembre de 2010. Comunicación de la necesidad de corrección: 4 de marzo de 2011. Recepción del documento corregido: 8 de marzo de 2011. Aceptado (con indicación de cambios): 18 de junio de 2011. Recepción del artículo corregido: 21 de junio de 2011.] 